\title{
SEMIGROUPS OF LINEAR TRANSFORMATIONS WITH RESTRICTED RANGE
}

\author{
R. P. SULLIVAN
}

(Received 15 August 2007)

\begin{abstract}
In 1975, Symons described the automorphisms of the semigroup $T(X, Y)$ consisting of all total transformations from a set $X$ into a fixed subset $Y$ of $X$. Recently Sanwong, Singha and Sullivan determined all maximal (and all minimal) congruences on $T(X, Y)$, and Sommanee studied Green's relations in $T(X, Y)$. Here, we describe Green's relations and ideals for the semigroup $T(V, W)$ consisting of all linear transformations from a vector space $V$ into a fixed subspace $W$ of $V$.
\end{abstract}

2000 Mathematics subject classification: primary 20M20; secondary 15A04.

Keywords and phrases: linear transformation, Green's relations, ideals.

\section{Introduction}

If $X$ is a set, we let $T(X)$ denote the semigroup (under composition) of all total transformations of $X$. In addition, if $Y \subseteq X$, we let $X \alpha=\operatorname{ran} \alpha$ denote the range of $\alpha$ and write

$$
T(X, Y)=\{\alpha \in T(X) \mid X \alpha \subseteq Y\} .
$$

This is a subsemigroup of $T(X)$. In fact, if $|Y|=1$ then $T(X, Y)$ contains exactly one element (namely, the constant mapping with range $Y$ ).

In 1975, Symons [6] described all the automorphisms of $T(X, Y)$ : this is an elegant and significant result and, surprisingly, it depends on whether $Y$ contains exactly two, or more than two, elements (the former case is the much harder one to resolve). In [3] the authors characterized the regular elements in $T(X, Y)$, and all maximal (and all minimal) congruences on $T(X, Y)$ were described in [4]. Also, in [5] Sommanee studied Green's relations in $T(X, Y)$. Here we describe Green's relations and ideals for the semigroup $T(V, W)$ consisting of all linear transformations from a vector space $V$ into a fixed subspace $W$ of $V$. As a consequence, we show that $T(V, W)$ is almost never isomorphic to $T(U)$ for any vector space $U$, and thus it is worth studying the algebraic properties of the semigroup $T(V, W)$ in its own right.

(c) 2008 Australian Mathematical Society 0004-9727/08 \$A2.00+0.00 


\section{Green's relations on $T(V, W)$}

Suppose that $W$ is a nonzero proper subspace of a vector space $V$, and let $T(V)$ denote the semigroup (under composition) of all linear mappings from $V$ into itself. Our aim in this section is to consider properties of the subsemigroup of $T(V)$ defined by

$$
T(V, W)=\{\alpha \in T(V) \mid V \alpha \subseteq W\} .
$$

To do this, we need some notation. For each $\alpha \in T(V)$, we write $\operatorname{ker} \alpha$ and $V \alpha=\operatorname{ran} \alpha$ for the kernel and the range of $\alpha$, respectively, and we write

$$
n(\alpha)=\operatorname{dim}(\operatorname{ker} \alpha) \quad \text { and } \quad r(\alpha)=\operatorname{dim}(\operatorname{ran} \alpha) .
$$

As an abbreviation, we write a subset $\left\{e_{i} \mid i \in I\right\}$ of $V$ as $\left\{e_{i}\right\}$, letting the subscript denote an (unspecified) index set $I$ (this is comparable with [1, Volume 2, p. 241]). We write the subspace of $V$ generated by a linearly independent subset $\left\{e_{i}\right\}$ of $V$ as $\left\langle e_{i}\right\rangle$; and, when we write $U=\left\langle e_{i}\right\rangle$, we tacitly assume that the set $\left\{e_{i}\right\}$ is a basis for the subspace $U$.

Often it is necessary to construct some $\alpha \in T(V)$ by first choosing a basis $\left\{e_{i}\right\}$ for $V$ and some subset $\left\{u_{i}\right\}$ of $V$, and then letting $e_{i} \alpha=u_{i}$ for each $i \in I$ and extending this action by linearity to the whole of $V$. To abbreviate matters, we simply say, given $\left\{e_{i}\right\}$ and $\left\{u_{i}\right\}$ within context, that $\alpha \in T(V)$ is defined by letting

$$
\alpha=\left(\begin{array}{c}
e_{i} \\
u_{i}
\end{array}\right) \text {. }
$$

To characterize Green's relations on $T(V, W)$, we need to describe all of its regular elements. This was done in [2, Theorem 2.1], but we include a proof for completeness.

LEMMA 1. The set $Q$ of all regular elements in $T(V, W)$ forms a semigroup and is given by

$$
Q=\{\alpha \in T(V, W) \mid V \alpha \subseteq W \alpha\} .
$$

Proof. Clearly, if $\alpha \in Q$ and $\beta \in T(V)$, then $V \alpha \subseteq W \alpha$ implies that $V \alpha \beta \subseteq W \alpha \beta$, so $Q$ is a right ideal of $T(V)$ and, in particular, it is a subsemigroup of $T(V, W)$. Suppose that $\alpha=\alpha \beta \alpha$ for some $\beta \in T(V, W)$. Then $u \alpha=(u \alpha \beta) \alpha \in W \alpha$ for all $u \in V$, so $V \alpha \subseteq W \alpha$ and hence $\alpha \in Q$.

Conversely, suppose that $\alpha \in T(V, W)$ and $V \alpha \subseteq W \alpha=\left\langle w_{j} \alpha\right\rangle$, where $w_{j} \in W$ for each $j$. Then $\left\{w_{j}\right\}$ is linearly independent. Also, if $v \in V$ then $v \alpha=\left(\sum x_{j} w_{j}\right) \alpha$ for some scalars $x_{j}$, and so $V=\operatorname{ker} \alpha \oplus\left\langle w_{j}\right\rangle$. If $\operatorname{ker} \alpha=\left\langle u_{i}\right\rangle$ and $V=W \alpha \oplus\left\langle v_{k}\right\rangle$, we can write

$$
\alpha=\left(\begin{array}{cc}
u_{i} & w_{j} \\
0 & w_{j} \alpha
\end{array}\right), \quad \beta=\left(\begin{array}{cc}
v_{k} & w_{j} \alpha \\
0 & w_{j}
\end{array}\right),
$$

and observe that $V \beta=\left\langle w_{j}\right\rangle \subseteq W$, so $\beta \in T(V, W)$ and $\alpha=\alpha \beta \alpha$. 
Note that $Q$ is always nonempty: if $W=V$ then $Q=T(V)$ which is regular for all vector spaces $V$ (see [1, Volume 1, Exercise 2.2.6]); if $W=\{0\}$ then $Q$ contains only the zero mapping in $T(V)$, and hence it is trivially regular; and if $W=\left\langle w_{j}\right\rangle$ and $V=\left\langle u_{i}\right\rangle \oplus W$ then clearly

$$
\alpha=\left(\begin{array}{cc}
u_{i} & w_{j} \\
0 & w_{j}
\end{array}\right) \in Q .
$$

In addition, although $Q$ is always a right ideal of $T(V, W)$, it is almost never a left ideal. For example, if $W=\langle w\rangle$ and $V=\langle v, w\rangle$ and

$$
\alpha=\left(\begin{array}{cc}
v & w \\
0 & w
\end{array}\right), \quad \lambda=\left(\begin{array}{cc}
w & v \\
0 & w
\end{array}\right),
$$

then $\alpha \in Q$ but $\lambda \alpha=\lambda \notin Q$.

LemMA 2. Let $\gamma \in Q$ and $\beta \in T(V, W)$. Then $\beta=\lambda \gamma$ for some $\lambda \in T(V, W)$ if and only if $\operatorname{ran} \beta \subseteq \operatorname{ran} \gamma$. Consequently, if $\alpha, \beta \in T(V, W)$ then $\alpha \mathcal{L} \beta$ in $T(V, W)$ if and only if $\alpha=\beta$ or $(\operatorname{ran} \alpha=\operatorname{ran} \beta$ and $\alpha, \beta \in Q)$.

Proof. Clearly, if $\beta=\lambda \gamma$ for some $\lambda \in T(V, W)$ then $\operatorname{ran} \beta \subseteq \operatorname{ran} \gamma$. Conversely, suppose that $\operatorname{ran} \beta \subseteq \operatorname{ran} \gamma \subseteq W \gamma$ and write $\operatorname{ran} \beta=\left\langle v_{i} \beta\right\rangle$. Then, for each $i$, there exists $w_{i} \in W$ such that $v_{i} \beta=w_{i} \gamma$ and we let $\operatorname{ran} \gamma=\left\langle w_{i} \gamma\right\rangle \oplus\left\langle w_{j} \gamma\right\rangle$. Note that, if $\operatorname{ker} \beta=\left\langle u_{r}\right\rangle$ and $\operatorname{ker} \gamma=\left\langle u_{s}\right\rangle$, then both $\left\{u_{r}\right\} \cup\left\{v_{i}\right\}$ and $\left\{u_{s}\right\} \cup\left\{w_{i}\right\} \cup\left\{w_{j}\right\}$ are linearly independent. Also, $V=\operatorname{ker} \beta \oplus\left\langle v_{i}\right\rangle$ and $V=\operatorname{ker} \gamma \oplus\left\langle w_{i}\right\rangle \oplus\left\langle w_{j}\right\rangle$. Thus, we can write

$$
\beta=\left(\begin{array}{cc}
u_{r} & v_{i} \\
0 & w_{i} \gamma
\end{array}\right), \quad \gamma=\left(\begin{array}{ccc}
u_{s} & w_{i} & w_{j} \\
0 & w_{i} \gamma & w_{j} \gamma
\end{array}\right),
$$

and define $\lambda \in T(V, W)$ by

$$
\lambda=\left(\begin{array}{cc}
u_{r} & v_{i} \\
0 & w_{i}
\end{array}\right) .
$$

Then $\beta=\lambda \gamma$, as required. Now suppose that $\alpha \mathcal{L} \beta$ in $T(V, W)$, so $\alpha=\lambda \beta$ and $\beta=\lambda^{\prime} \alpha$ for some $\lambda, \lambda^{\prime} \in T(V, W)^{1}$. If $\lambda=1$ or $\lambda^{\prime}=1$ then $\alpha=\beta$. On the other hand, if $\lambda, \lambda^{\prime} \neq 1$ then $\lambda, \lambda^{\prime} \in T(V, W)$ and

$$
\alpha=\lambda \lambda^{\prime} \cdot \alpha \text { and } \beta=\lambda^{\prime} \lambda \cdot \beta .
$$

Hence, $V \alpha=\left(V \lambda \lambda^{\prime}\right) \alpha \subseteq W \alpha$, and similarly $V \beta \subseteq W \beta$, so $\alpha, \beta \in Q$, and clearly $\operatorname{ran} \alpha=\operatorname{ran} \beta$. The converse is clear by the first part of the lemma.

LEMMA 3. If $\alpha, \beta \in T(V, W)$, then $\beta=\alpha \mu$ for some $\mu \in T(V, W)$ if and only if $\operatorname{ker} \alpha \subseteq \operatorname{ker} \beta$. Consequently, $\alpha \mathcal{R} \beta$ in $T(V, W)$ if and only if $\operatorname{ker} \alpha=\operatorname{ker} \beta$.

PROOF. Clearly, if $\beta=\alpha \mu$ for some $\mu \in T(V, W)$, then $\operatorname{ker} \alpha \subseteq \operatorname{ker} \beta$. Conversely, suppose that $\operatorname{ker} \alpha \subseteq \operatorname{ker} \beta$. Write $\operatorname{ker} \alpha=\left\langle u_{i}\right\rangle, \operatorname{ker} \beta=\left\langle u_{i}, u_{j}\right\rangle$ and $V=\operatorname{ker} \beta$ $\oplus\left\langle v_{k}\right\rangle$. Then

$$
\alpha=\left(\begin{array}{ccc}
u_{i} & u_{j} & v_{k} \\
0 & w_{j} & w_{k}
\end{array}\right), \quad \beta=\left(\begin{array}{ccc}
u_{i} & u_{j} & v_{k} \\
0 & 0 & w_{k}^{\prime}
\end{array}\right),
$$


for some $w_{j}, w_{k}, w_{k}^{\prime} \in W$. Let $V=\operatorname{ran} \alpha \oplus\left\langle v_{\ell}\right\rangle$ and define $\mu \in T(V, W)$ by

$$
\mu=\left(\begin{array}{ccc}
v_{\ell} & w_{j} & w_{k} \\
0 & 0 & w_{k}^{\prime}
\end{array}\right)
$$

Then $\beta=\alpha \mu$, as required, and the remaining assertion is clear.

Note that, if $\alpha \in T(V, W)$, then $\operatorname{dim}(W \alpha) \leq r(\alpha)$. Also, recall that the rank-nullity theorem for arbitrary vector spaces can be proved by showing that, for each $\pi \in T(V)$, the mapping

$$
V \pi \rightarrow V / \operatorname{ker} \pi, \quad v \pi \rightarrow v+\operatorname{ker} \pi
$$

is a well-defined (vector space) isomorphism. Hence, if $\operatorname{ker} \beta \subseteq \operatorname{ker} \alpha$, then $r(\beta) \geq r(\alpha)$.

We also need to observe that if $\alpha \in T(V, W)$, and we write $W \cap \operatorname{ker} \alpha=\left\langle u_{i}\right\rangle$ and $W=\left\langle u_{i}\right\rangle \oplus\left\langle u_{j}\right\rangle$, then $\operatorname{dim}(W \alpha)=|J|$. This follows from the fact that $\left\{u_{j} \alpha\right\}$ is a basis for $W \alpha$, and the restriction $\alpha \mid\left\langle u_{j}\right\rangle$ is a (vector space) isomorphism from $\left\langle u_{j}\right\rangle$ onto $W \alpha$.

LEMMA 4. If $\alpha, \beta \in T(V, W)$, then $\beta=\lambda \alpha \mu$ for some $\lambda, \mu \in T(V, W)$ if and only if $r(\beta) \leq \operatorname{dim}(W \alpha)$. Consequently, $\alpha \mathcal{J} \beta$ in $T(V, W)$ if and only if one of the following equalities occurs:

(J1) $\operatorname{ker} \alpha=\operatorname{ker} \beta$;

(J2) $r(\alpha)=\operatorname{dim}(W \alpha)=\operatorname{dim}(W \beta)=r(\beta)$.

PROOF. If $\beta=\lambda \alpha \mu$ for some $\lambda, \mu \in T(V, W)$, then $V \beta=(V \lambda) \alpha \mu \subseteq(W \alpha) \mu$, so $\operatorname{dim}(V \beta) \leq \operatorname{dim}(W \alpha)$. Conversely, suppose that the condition holds and write

$$
\beta=\left(\begin{array}{cc}
u_{i} & v_{k} \\
0 & w_{k}
\end{array}\right), \quad \alpha=\left(\begin{array}{ccc}
u_{j} & w_{k}^{\prime} & v_{\ell} \\
0 & w_{k}^{\prime} \alpha & w_{\ell}
\end{array}\right),
$$

where $V \beta=\left\langle w_{k}\right\rangle,\left\langle w_{k}^{\prime}\right\rangle \subseteq W$ and $V \alpha=\left\langle w_{k}^{\prime} \alpha\right\rangle \oplus\left\langle w_{\ell}\right\rangle \subseteq W$. Let $V=\left\langle w_{k}^{\prime} \alpha\right\rangle \oplus\left\langle v_{m}\right\rangle$ and define $\lambda, \mu \in T(V, W)$ by

$$
\lambda=\left(\begin{array}{cc}
u_{i} & v_{k} \\
0 & w_{k}^{\prime}
\end{array}\right), \quad \mu=\left(\begin{array}{cc}
v_{m} & w_{k}^{\prime} \alpha \\
0 & w_{k}
\end{array}\right) .
$$

Then $\beta=\lambda \alpha \mu$, as required.

Now, suppose that $\beta=\lambda \alpha \mu$ and $\alpha=\lambda^{\prime} \beta \mu^{\prime}$ for some $\lambda, \lambda^{\prime}, \mu, \mu^{\prime} \in T(V, W)^{1}$. If $\lambda=1$ then $\operatorname{ker} \alpha \subseteq \operatorname{ker} \beta$; and if $\lambda \neq 1$ then $r(\beta)=\operatorname{dim}(V \lambda \alpha) \mu \leq \operatorname{dim}(W \alpha)$. In other words, the supposition implies that

$$
\begin{array}{lll}
\operatorname{ker} \alpha \subseteq \operatorname{ker} \beta & \text { or } \quad r(\beta) \leq \operatorname{dim}(W \alpha) \quad \text { and } \\
\operatorname{ker} \beta \subseteq \operatorname{ker} \alpha & \text { or } \quad r(\alpha) \leq \operatorname{dim}(W \beta),
\end{array}
$$

and the different combinations give the following possibilities: 
(J1) $\operatorname{ker} \alpha=\operatorname{ker} \beta$;

(J2) $r(\alpha)=\operatorname{dim}(W \alpha)=\operatorname{dim}(W \beta)=r(\beta)$;

(J3) $\operatorname{ker} \alpha \subseteq \operatorname{ker} \beta$ and $r(\alpha) \leq \operatorname{dim}(W \beta)$;

(J4) $\operatorname{ker} \beta \subseteq \operatorname{ker} \alpha$ and $r(\beta) \leq \operatorname{dim}(W \alpha)$.

However, if (J3) occurs then $W \cap \operatorname{ker} \alpha \subseteq W \cap \operatorname{ker} \beta$ and $r(\alpha) \geq r(\beta)$. Hence, if

$W \cap \operatorname{ker} \beta=(W \cap \operatorname{ker} \alpha) \oplus U_{1} \quad$ and $\quad W=(W \cap \operatorname{ker} \beta) \oplus U_{2}$,

then $W \alpha=U_{1} \alpha \oplus U_{2} \alpha$ and $W \beta=U_{2} \beta$. Consequently,

$$
\begin{aligned}
\operatorname{dim}(W \beta) & =\operatorname{dim} U_{2}=\operatorname{dim}\left(U_{2} \alpha\right) \leq \operatorname{dim}(W \alpha), \\
\operatorname{dim}(W \alpha) & \leq r(\alpha) \leq \operatorname{dim}(W \beta) \quad \text { and } \\
r(\beta) & \leq r(\alpha) \leq \operatorname{dim}(W \beta) \leq r(\beta) .
\end{aligned}
$$

It follows that (J2) holds, and similarly, (J4) also implies (J2). For the converse, recall that $\mathcal{R} \subseteq \mathcal{J}$. Hence, if either $(\mathrm{J} 1)$ or $(\mathrm{J} 2)$ occurs then Lemma 3, and the first part of this lemma, imply that $\alpha \mathcal{J} \beta$.

From Lemma 4, we see that, if $\alpha \mathcal{J} \beta$, then $r(\alpha)=r(\beta)$. However, the converse is false, even if $V$ has finite dimension. This differs from the situation for $T(V)$ and arbitrary $V$, since it is well known that $\alpha \mathcal{J} \beta$ in $T(V)$ if and only if $r(\alpha)=r(\beta)$ : see [1, Volume 1, Exercise 2.2.6].

EXAMPLE 1. Let $V=\left\langle e_{1}, e_{2}, e_{3}\right\rangle$ and $W=\left\langle e_{1}, e_{2}\right\rangle$, and define $\alpha, \beta \in T(V, W)$ by

$$
\alpha=\left(\begin{array}{ccc}
e_{1} & e_{2} & e_{3} \\
0 & e_{2} & e_{1}
\end{array}\right), \quad \beta=\left(\begin{array}{ccc}
e_{1} & e_{2} & e_{3} \\
e_{1} & 0 & e_{2}
\end{array}\right) .
$$

Then $\operatorname{ker} \alpha \neq \operatorname{ker} \beta$, so (J1) does not hold. Also, $r(\alpha)=r(\beta)=2$, but $\operatorname{dim}(W \alpha)$ $=\operatorname{dim}(W \beta)=1$, so $(\mathrm{J} 2)$ does not hold. Hence, $\alpha$ and $\beta$ are not $\mathcal{J}$-related in $T(V, W)$. Furthermore, $V \alpha \nsubseteq W \alpha$ and $V \beta \nsubseteq W \beta$, so $\alpha, \beta \notin Q$. Hence, $\alpha, \beta$ are also not $\mathcal{L}$ related in $T(V, W)$, even though $\operatorname{ran} \alpha=\operatorname{ran} \beta$.

In fact, this example shows more: namely, even though $r(\beta)=r(\alpha)$ and $\operatorname{dim}(W \beta)=\operatorname{dim}(W \alpha)$ for the given $\alpha$ and $\beta$, nonetheless $\beta \neq \lambda \alpha \mu$ for all $\lambda, \mu$ $\in T(V, W)$. This is unlike the situation in $T(V)$, where $\beta=\lambda \alpha \mu$ for some $\lambda, \mu$ $\in T(V)$ if and only if $\operatorname{dim}(V \beta) \leq \operatorname{dim}(V \alpha)$. However , by restricting our attention to $Q$, we regain the normal situation.

LEMMA 5. If $\alpha, \beta \in Q$, then $\beta=\lambda \alpha \mu$ for some $\lambda, \mu \in Q$ if and only if $r(\beta)$ $\leq r(\alpha)$. Consequently, $\alpha \mathcal{J} \beta$ in $Q$ if and only if $r(\alpha)=r(\beta)$, and hence $\mathcal{J}=\mathcal{D}$ on $Q$.

PROOF. Clearly, the given condition implies that $r(\beta) \leq r(\alpha)$. Conversely, suppose that $\alpha, \beta \in Q$ and $r(\beta) \leq r(\alpha)$. Then $V \alpha=W \alpha$ and $V \beta=W \beta$, so we can write

$$
\beta=\left(\begin{array}{cc}
u_{r} & w_{i} \\
0 & w_{i} \beta
\end{array}\right), \quad \alpha=\left(\begin{array}{ccc}
u_{s} & w_{i}^{\prime} & w_{j} \\
0 & w_{i}^{\prime} \alpha & w_{j} \alpha
\end{array}\right) .
$$


Now let $V=\left\langle u_{t}\right\rangle \oplus\left\langle w_{i}^{\prime} \alpha\right\rangle$ and define $\lambda, \mu \in T(V, W)$ by

$$
\lambda=\left(\begin{array}{cc}
u_{r} & w_{i} \\
0 & w_{i}^{\prime}
\end{array}\right), \quad \mu=\left(\begin{array}{cc}
v_{t} & w_{i}^{\prime} \alpha \\
0 & w_{i} \beta
\end{array}\right) .
$$

Then $\beta=\lambda \alpha \mu$. Moreover, since $V=\left\langle u_{r}\right\rangle \oplus\left\langle w_{i}\right\rangle$, we know that $V \lambda=\left\langle w_{i} \lambda\right\rangle \subseteq W \lambda$, and so $\lambda \in Q$. Similarly, $V \mu=\left\langle\left(w_{i}^{\prime} \alpha\right) \mu\right\rangle \subseteq W \mu$ (since $w_{i}^{\prime} \alpha \in W$ for each $i$ ) and hence $\mu \in Q$.

Finally, if $\beta=\lambda \alpha \mu$ and $\alpha=\lambda^{\prime} \beta \mu^{\prime}$ for some $\lambda, \lambda^{\prime}, \mu, \mu^{\prime} \in Q^{1}$ then, regardless of whether $\lambda=1$ or $\mu=1$,

$$
\operatorname{dim}(V \beta)=\operatorname{dim}(V \lambda) \alpha \mu \leq \operatorname{dim}(V \alpha) \mu \leq \operatorname{dim}(V \alpha) .
$$

That is, $r(\beta) \leq r(\alpha)$, and similarly $r(\alpha) \leq r(\beta)$. The converse is clear from the first part of this lemma. Finally, since $Q$ is a regular subsemigroup of $T(V, W)$, T. E. Hall's theorem allows us to deduce that the $\mathcal{L}$ and $\mathcal{R}$ relations on $Q$ are the restrictions of those on $T(V, W)$ to $Q$. Thus, by Lemmas 2 and 3, if $\alpha, \beta \in Q$ then $\alpha \mathcal{L} \beta$ in $Q$ if and only if $\operatorname{ran} \alpha=\operatorname{ran} \beta$, and $\alpha \mathcal{R} \beta$ in $Q$ if and only if $\operatorname{ker} \alpha=\operatorname{ker} \beta$. Consequently, a standard argument shows that, if $r(\alpha)=r(\beta)$, then $\alpha \mathcal{D} \beta$, and we conclude that $\mathcal{J}=\mathcal{D}$ on $Q$.

Lemma 6. If $\alpha, \beta \in T(V, W)$, then $\alpha \mathcal{D} \beta$ in $T(V, W)$ if and only if either ker $\alpha$ $=\operatorname{ker} \beta$ or $(r(\alpha)=r(\beta)$ and $\alpha, \beta \in Q)$.

Proof. If $\alpha \mathcal{D} \beta$ in $T(V, W)$, then $\alpha \mathcal{R} \gamma \mathcal{L} \beta$ for some $\gamma \in T(V, W)$. Hence, $\operatorname{ker} \alpha=\operatorname{ker} \gamma$, and either $\gamma=\beta$ or $(\operatorname{ran} \gamma=\operatorname{ran} \beta$ and $\gamma, \beta \in Q)$. If $\operatorname{ker} \alpha=\operatorname{ker} \gamma$ and $\gamma=\beta$ then $\operatorname{ker} \alpha=\operatorname{ker} \beta$, as required. On the other hand, suppose that ker $\alpha=\operatorname{ker} \gamma$, $\operatorname{ran} \gamma=\operatorname{ran} \beta$ and $\gamma, \beta \in Q$. Then $\alpha=\gamma \mu$ for some $\mu \in T(V, W)$, so $V \gamma \subseteq W \gamma$ implies that $V \alpha \subseteq W \alpha$, and hence $\alpha \in Q$. Similarly, $\beta \in Q$. Also,

$$
r(\beta)=r(\gamma)=\operatorname{dim}(V / \operatorname{ker} \gamma)=\operatorname{dim}(V / \operatorname{ker} \alpha)=r(\alpha) .
$$

Conversely, if $\operatorname{ker} \alpha=\operatorname{ker} \beta$ then $\alpha \mathcal{R} \beta$, and so $\alpha \mathcal{D} \beta$ (since $\mathcal{R} \subseteq \mathcal{D}$ ). On the other hand, if $\alpha, \beta \in Q$ and $r(\alpha)=r(\beta)$, then $V \alpha=W \alpha$ and $V \beta=W \beta$, so we can write

$$
\alpha=\left(\begin{array}{cc}
u_{r} & w_{j} \\
0 & w_{j} \alpha
\end{array}\right), \quad \beta=\left(\begin{array}{cc}
u_{s} & w_{j}^{\prime} \\
0 & w_{j}^{\prime} \beta
\end{array}\right),
$$

where $\left\langle w_{j}\right\rangle \subseteq W$ and $\left\langle w_{j}^{\prime}\right\rangle \subseteq W$. If $\gamma \in T(V, W)$ is defined by

$$
\gamma=\left(\begin{array}{cc}
u_{r} & w_{j} \\
0 & w_{j}^{\prime} \beta
\end{array}\right),
$$

then $\operatorname{ker} \gamma=\operatorname{ker} \alpha, \operatorname{ran} \gamma=\operatorname{ran} \beta$ and $\gamma \in Q$, so $\alpha \mathcal{R} \gamma \mathcal{L} \beta$. 
Recall that $\mathcal{D} \subseteq \mathcal{J}$ on any semigroup, and it is well known that $\mathcal{D}=\mathcal{J}$ on any $T(V)$ (see [1, Volume 1, Exercise 2.2.6]). However, this fails for $T(V, W)$, as we now show.

EXAmple 2. If $\alpha \mathcal{D} \beta$ in $T(V, W)$ then either $\operatorname{ker} \alpha=\operatorname{ker} \beta$ (so (J1) holds) or $r(\alpha)=r(\beta)$ and $\alpha, \beta \in Q$ (hence $\operatorname{dim}(W \alpha)=\operatorname{dim}(W \beta)$ and (J2) holds). However, $\mathcal{J} \backslash \mathcal{D}$ can be nonempty. For example, suppose that $V=\left\langle u_{0}, u_{1}, u_{2}, w_{1}, w_{k}\right\rangle$ and $W=\left\langle w_{1}, w_{k}\right\rangle$, where $K$ is infinite. In this event, we can define $\alpha, \beta \in T(V, W)$ by

$$
\alpha=\left(\begin{array}{ccc}
u_{0} & u_{1} & \left\{u_{2}, w_{1}, w_{k}\right\} \\
0 & w_{1} & w_{k}
\end{array}\right), \quad \beta=\left(\begin{array}{cccc}
u_{0} & u_{1} & u_{2} & \left\{w_{1}, w_{k}\right\} \\
0 & 0 & w_{1} & w_{k}
\end{array}\right) .
$$

Then $u_{2}-w_{1} \in \operatorname{ker} \alpha$, so $\operatorname{ker} \alpha \subsetneq \operatorname{ker} \beta$; and $r(\alpha)=|K|=\operatorname{dim}(W \beta)$, so $\alpha, \beta$ satisfy (J2). But, although $r(\alpha)=r(\beta)$, we observe that $W \alpha=\left\langle w_{k}\right\rangle=W \beta$, so $W \alpha \subsetneq V \alpha$ and $W \beta \subsetneq V \beta$. Therefore, $\alpha, \beta \notin Q$, and hence $\alpha, \beta$ are not $\mathcal{D}$-related in $T(V, W)$.

Corollary 7. If $\operatorname{dim} W<\aleph_{0}$ then $\mathcal{D}=\mathcal{J}$ on $T(V, W)$.

ProOf. Suppose that $\alpha, \beta \in T(V, W)$ and $\alpha \mathcal{J} \beta$. By Lemma 3, if ker $\alpha=\operatorname{ker} \beta$ then $\alpha \mathcal{D} \beta$; and, by Lemma 4 , if $\operatorname{ker} \alpha \neq \operatorname{ker} \beta$ then $r(\alpha)=\operatorname{dim}(W \alpha)=\operatorname{dim}(W \beta)=r(\beta)$. Consequently, in this case, if $\operatorname{dim} W<\aleph_{0}$ then $r(\alpha), r(\beta)<\aleph_{0}$, and it follows that $V \alpha=W \alpha$ and $V \beta=W \beta$. Thus, $\alpha, \beta \in Q$ and $r(\alpha)=r(\beta)$, so $\alpha \mathcal{D} \beta$.

\section{Ideals in $T(V, W)$}

In what follows, $Y=A \dot{\cup} B$ means that $Y$ is a disjoint union of $A$ and $B$, and $r^{\prime}$ denotes the successor of a cardinal $r$. Also, as an abbreviation, we sometimes write $T=T(V, W)$.

As might be expected, the ideals of $Q$ are easy to describe.

THEOREM 8. The ideals of $Q$ are precisely the sets

$$
Q_{r}=\{\alpha \in Q \mid r(\alpha)<r\},
$$

where $1 \leq r \leq \operatorname{dim} W$. In addition, $Q_{r}$ is principal if and only if $r=s^{\prime}$, where $1 \leq s \leq \operatorname{dim} W$.

PRoOF. If $\alpha \in Q_{r}$ and $\beta \in Q$, then $\operatorname{dim}(V \alpha) \beta \leq \operatorname{dim}(V \alpha)$ and $V(\beta \alpha) \subseteq V \alpha$, so $\alpha \beta \in Q_{r}$ and $\beta \alpha \in Q_{r}$, and hence $Q_{r}$ is an ideal of $Q$. Conversely, suppose that $I$ is an ideal of $Q$ and let $r$ be the least cardinal greater than $r(\alpha)$ for all $\alpha \in I$. Then $I \subseteq Q_{r}$. Let $\beta \in Q_{r}$ and suppose that $r(\beta)=s<r$. Then there exists $\alpha \in I$ with $r(\alpha) \geq s$ : otherwise, $r(\alpha)<s$ for all $\alpha \in I$, contradicting the choice of $r$. That is, $r(\beta) \leq r(\alpha)$, and Lemma 5 implies that $\beta=\lambda \alpha \mu$ for some $\lambda, \mu \in Q$. That is, $Q_{r} \subseteq I$, and equality follows.

Finally, if $r=s^{\prime}$ for some $s$ such that $1 \leq s \leq \operatorname{dim} W$, then Lemma 5 implies that $Q_{r} \subseteq Q^{1} \alpha Q^{1}$ for each $\alpha \in Q_{r}$ with rank $s$, and it follows that $Q_{r}$ is principal. 
Conversely, suppose that $Q_{r}=Q^{1} \alpha Q^{1}$ for some $\alpha \in Q_{r}$. Let $r(\alpha)=s$ and assume there is a cardinal $t$ such that $s<t<r$. Since $r \leq \operatorname{dim} W$, there exists $\beta \in Q$ with $r(\beta)=t$. For example, we can write $W=\left\langle e_{i}, e_{j}\right\rangle$ and $V=\left\langle e_{i}, e_{j}, e_{k}\right\rangle$ where $|I|=t$, and let

$$
\beta=\left(\begin{array}{cc}
\left\{e_{j}, e_{k}\right\} & e_{i} \\
0 & e_{i}
\end{array}\right)
$$

Now $\beta \in Q_{r}$, so $\beta=\lambda \alpha \mu$ for some $\lambda, \mu \in Q^{1}$. But this implies that $r(\beta) \leq r(\alpha)$, which is a contradiction. Therefore, $t$ does not exist and thus $r=s^{\prime}$.

To determine the ideals of $T=T(V, W)$, we let $1 \leq r \leq \operatorname{dim} W$ and write

$$
T_{r}=\{\alpha \in T \mid r(\alpha)<r\} .
$$

If $\alpha \in T_{r}$ and $\lambda, \mu \in T$ then $V \lambda \alpha \subseteq V \alpha$ and $\operatorname{dim}(V \alpha \mu) \leq \operatorname{dim} V \alpha$, so $T_{r}$ is an ideal of $T$.

Let $Y \subseteq T(V, W)$ be nonempty and let

$$
\begin{gathered}
r(Y)=\min \{r \mid r>\operatorname{dim}(W \alpha) \text { for all } \alpha \in Y\}, \\
K(Y)=\{\beta \in T(V, W) \mid \operatorname{ker} \beta \supseteq \operatorname{ker} \alpha \text { for some } \alpha \in Y\} .
\end{gathered}
$$

Note that $r(Y)$ always exists since the cardinals are well ordered.

LEMMA 9. With the above notation, both $T_{r(Y)} \cup K(Y)$ and $T_{r(Y)^{\prime}} \cup K(Y)$ are ideals of $T(V, W)$.

Proof. Since $\operatorname{ker} \beta \subseteq \operatorname{ker} \beta \mu$ for each $\mu \in T(V, W), K(Y)$ is a right ideal of $T(V, W)$. On the other hand, if $\lambda \in T(V, W)$ and $\beta \in K(Y)$ then Lemma 3 implies that $\beta=\alpha \mu$ for some $\alpha \in Y$ and $\mu \in T(V, W)$, hence

$$
\operatorname{dim}(V \lambda \beta) \leq \operatorname{dim}(W \beta)=\operatorname{dim}(W \alpha \mu) \leq \operatorname{dim}(W \alpha)<r(Y) .
$$

Therefore $\lambda \beta \in T_{r(Y)}$. The result now follows since $T_{r(Y)}$ and $T_{r(Y)^{\prime}}$ are themselves ideals of $T(V, W)$.

EXAMPLE 3. Let $V=\left\langle u_{1}, u_{2}, u_{3}, w_{1}, w_{2}, w_{3}\right\rangle, W=\left\langle w_{1}, w_{2}, w_{3}\right\rangle$ and

$$
\alpha=\left(\begin{array}{cccc}
u_{1} & \left\{w_{1}, w_{2}, w_{3}\right\} & u_{2} & u_{3} \\
0 & 0 & w_{2} & w_{3}
\end{array}\right) .
$$

If $T=T(V, W)$ then $\alpha T$ is not only a right ideal but also a left ideal of $T$ since $\lambda \alpha=0$ for each $\lambda \in T$. Let $Y=\{\alpha\}$. Then $r(Y)=1$ and $T_{r(Y)}=\{0\}$, and clearly $\alpha T=K(\{\alpha\})$. 
EXAMPLE 4. Let $V=\left\langle u_{1}, u_{2}, u_{3}, w_{1}, w_{2}, w_{3}\right\rangle, W=\left\langle w_{1}, w_{2}, w_{3}\right\rangle$ and

$$
\begin{gathered}
\alpha=\left(\begin{array}{ccccc}
u_{1} & \left\{w_{2}, w_{3}\right\} & w_{1} & u_{2} & u_{3} \\
0 & 0 & w_{1} & w_{2} & w_{3}
\end{array}\right), \\
\lambda_{1}=\left(\begin{array}{cccc}
\left\{w_{1}, w_{2}, w_{3}\right\} & u_{1} & u_{2} & u_{3} \\
0 & w_{1} & w_{2} & w_{3}
\end{array}\right) .
\end{gathered}
$$

Now $\alpha T$ is not a left ideal of $T$ since

$$
\lambda_{1} \alpha=\left(\begin{array}{ccc}
\left\{w_{1}, w_{2}, w_{3}\right\} & u_{1} & \left\{u_{2}, u_{3}\right\} \\
0 & w_{1} & 0
\end{array}\right) \notin \alpha T .
$$

Let $Y=\{\alpha\}$. Then $r(Y)=2$ and $T^{1} \alpha T^{1} \subseteq T_{2} \cup K(\{\alpha\})$ : for example, if $\lambda, \mu \in T$ then $\operatorname{dim}(V \lambda \alpha) \leq \operatorname{dim}(W \alpha)<2$ and $\alpha \mu \in K(\{\alpha\})$. In fact, if $\beta \in T_{2}$ then $\operatorname{dim}(V \beta)$ $\leq 1=\operatorname{dim}(W \alpha)$, so $\beta \in T \alpha T$ by Lemma 4. And, if $\beta \in K(\{\alpha\})$ then $\beta=\alpha \mu$ for some $\mu \in T$, so $\beta \in T^{1} \alpha T^{1}$. Hence $T^{1} \alpha T^{1}=T_{2} \cup K(\{\alpha\})$. On the other hand, $T \alpha T \subseteq T_{2}$ since $\operatorname{dim}(V \lambda \alpha \mu) \leq \operatorname{dim}(W \alpha) \mu \leq \operatorname{dim}(W \alpha)$ for all $\lambda, \mu \in T$, and $T_{2} \subseteq T \alpha T$ by Lemma 4 .

For our main result, we need a technical lemma.

LEMMA 10. If $\beta \in T$ and $r<\operatorname{dim}(W \beta)=\operatorname{dim}(V \beta)=s$, then there exists $\lambda \in T$ such that $\operatorname{dim}(W \lambda \beta)=r$ and $\operatorname{dim}(V \lambda \beta)=s$.

PROOF. If $s$ is finite then $W \beta=V \beta$, so we write

$$
\beta=\left(\begin{array}{ccccccc}
u_{p} & w_{1} & \ldots & w_{r} & w_{r+1} & \ldots & w_{s} \\
0 & w_{1}^{\prime} & \ldots & w_{r}^{\prime} & w_{r+1}^{\prime} & \ldots & w_{s}^{\prime}
\end{array}\right)
$$

Choose $u \in V \backslash W$ and note that $u+w_{j} \notin W$ for each $j=r+1, \ldots, s$. Also, the set $\left\{w_{1}, \ldots, w_{r}, u+w_{r+1}, \ldots, u+w_{s}\right\}$ is linearly independent: for example, if there are scalars such that

$$
\sum_{i=1}^{r} x_{i} w_{i}+\sum_{j=r+1}^{s} y_{j}\left(u+w_{j}\right)=0
$$

then $\sum_{i=1}^{r} x_{i} w_{i}+\sum_{j=r+1}^{s} y_{j} w_{j} \in\langle u\rangle$ and this implies that $x_{i}=y_{j}=0$ for each $i$ and $j$. Write $V=\left\langle u_{\ell}\right\rangle \oplus\left\langle w_{1}, \ldots, w_{r}, u+w_{r+1}, \ldots, u+w_{s}\right\rangle$ and let

$$
\lambda=\left(\begin{array}{ccccccc}
u_{\ell} & w_{1} & \ldots & w_{r} & u+w_{r+1} & \ldots & u+w_{s} \\
0 & w_{1} & \ldots & w_{r} & w_{r+1} & \ldots & w_{s}
\end{array}\right) .
$$

Then $\operatorname{dim}(W \lambda \beta)=r$ and $\operatorname{dim}(V \lambda \beta)=s$, as required.

If $s$ is infinite, write

$$
\beta=\left(\begin{array}{ccc}
u_{p} & w_{j} & v_{k} \\
0 & w_{j}^{\prime} & w_{k}
\end{array}\right)
$$


where $|J|+|K|=|J|=s \geq \aleph_{0}$. This implies that $|K| \leq|J|$, and clearly there exist $\lambda_{1} \in T$ and $u_{q} \in V$ such that

$$
\lambda_{1} \beta=\left(\begin{array}{cc}
u_{q} & w_{j} \\
0 & w_{j}^{\prime}
\end{array}\right) \in Q .
$$

Since $r<|J|$, we can write $J=M \dot{\cup} N$ where $|M|=r$ and $|N|=|J|$. Then, as before, if $u \in V \backslash W$ then $\left\{w_{m}\right\} \dot{\cup}\left\{u+w_{n}\right\}$ is linearly independent and we let

$$
\lambda_{2}=\left(\begin{array}{ccc}
u_{\ell} & w_{m} & u+w_{n} \\
0 & w_{m} & w_{n}
\end{array}\right) .
$$

Then $\operatorname{dim}\left(W \lambda_{2} \lambda_{1} \beta\right)=r$ and $\operatorname{dim}\left(V \lambda_{2} \lambda_{1} \beta\right)=s$, as required.

The proper ideals of $T(W)$ are well known: in fact, they are in one-to-one correspondence with the cardinals $r$ such that $1 \leq r \leq \operatorname{dim} W$ (see [1, Volume 1, Exercise 2.2.6]). However, the result for $T(V, W)$ is very different.

THEOREM 11. The ideals of $T(V, W)$ are precisely the sets $T_{r} \cup K(Y)$ and $T_{r^{\prime}} \cup K(Y)$, where $r=r(Y)$ and $Y$ is a nonempty subset of $T(V, W)$.

PRoOF. Let $I$ be an ideal of $T$. If $I=\{0\}$, we let $Y=I$, so $r(Y)=1, T_{1}=\{0\}$; and, if $\beta \in K(\{0\})$ then $\operatorname{ker} \beta=V$, so $\beta=0$ and thus $K(\{0\})=\{0\}$. That is, $\{0\}$ $=T_{1} \cup K(\{0\})$.

Suppose that $\alpha \in I$ is nonzero and write

$$
\alpha=\left(\begin{array}{ccc}
u_{p} & w_{j} & v_{k} \\
0 & w_{j}^{\prime} & w_{k}
\end{array}\right),
$$

where $v_{k} \notin W$ for each $k$. If $J=\emptyset$ then $K \neq \emptyset$ and $\operatorname{dim}(W \alpha)<\operatorname{dim}(V \alpha)$. On the other hand, if $J \neq \emptyset$, choose $1 \in J$ and $u \in V \backslash W$, write $V=\langle u\rangle \oplus\left\langle v_{\ell}\right\rangle$ where $W \subseteq\left\langle v_{\ell}\right\rangle$, and let

$$
\lambda=\left(\begin{array}{cc}
v_{\ell} & u \\
0 & w_{1}
\end{array}\right) .
$$

Then $\lambda \alpha \in I$ and $\operatorname{dim}(W \lambda \alpha)=0<1=\operatorname{dim}(V \lambda \alpha)$. That is, in each case, if

$$
Y=\{\alpha \in I \mid \operatorname{dim}(W \alpha)<\operatorname{dim}(V \alpha)\},
$$

then $Y \neq \emptyset$. We assert that $I$ equals $T_{r} \cup K(Y)$ or $T_{r^{\prime}} \cup K(Y)$, where $r=r(Y)$.

First suppose that $\operatorname{dim}(W \beta)<r$ for all $\beta \in I$. In this case, if $\beta \in I$ and $r(\beta)<r$ then $\beta \in T_{r}$ and, if $\operatorname{dim}(W \beta)<r \leq r(\beta)$, then $\beta \in Y$ and so $\beta \in K(Y)$. Thus, in this case, $I \subseteq T_{r} \cup K(Y)$. Conversely, suppose that $\beta \in T_{r}$. If $\operatorname{dim}(W \alpha)<r(\beta)<r$ for all $\alpha \in Y$, we contradict the choice of $r=r(Y)$. Hence, $r(\beta) \leq \operatorname{dim}(W \alpha)$ for some $\alpha \in Y \subseteq I$, hence $\beta \in I$ by Lemma 4. Clearly, $K(Y) \subseteq I$, so we conclude that $I=T_{r} \cup K(Y)$. 
Next suppose that $r \leq \operatorname{dim}(W \pi)$ for some $\pi \in I$. In this case, if $\operatorname{dim}(W \pi)$ $<\operatorname{dim}(V \pi)$ then $\pi \in Y$ and we contradict the choice of $r$. Hence $\operatorname{dim}(W \pi)$ $=\operatorname{dim}(V \pi)$. Now, if $r<\operatorname{dim}(W \pi)=\operatorname{dim}(V \pi)=s$, then Lemma 10 implies that $\operatorname{dim}(W \lambda \pi)=r<s=\operatorname{dim}(V \lambda \pi)$ for some $\lambda \in T$, which contradicts the choice of $r$ (since $\lambda \pi \in I$ ). Hence, in this case, $r=\operatorname{dim}(W \pi)=\operatorname{dim}(V \pi)$ and thus $\pi \in T_{r^{\prime}}$. Clearly this conclusion holds for any $\beta \in I$ such that $r \leq \operatorname{dim}(W \beta)$. On the other hand, if $\beta \in I$ and $\operatorname{dim}(W \beta)<r$, then we have already seen that $\beta \in T_{r} \cup K(Y)$. So, in this case, $I \subseteq T_{r^{\prime}} \cup K(Y)$. Conversely, if $\beta \in T_{r^{\prime}}$ then $r(\beta) \leq r=\operatorname{dim}(W \pi)=\operatorname{dim}(V \pi)$ for some $\pi \in I$, so $\beta \in I$ by Lemma 4. As before, $K(Y) \subseteq I$, and now we conclude that $I=T_{r^{\prime}} \cup K(Y)$.

EXAmple 5. Let $1 \leq r \leq \operatorname{dim} W$ and write

$$
J_{r}=\{\alpha \in T \mid \operatorname{dim}(W \alpha)<r\} .
$$

If $\alpha \in J_{r}$ and $\lambda, \mu \in T$, then $W \lambda \alpha \subseteq W \alpha$ and $\operatorname{dim}(W \alpha \mu) \leq \operatorname{dim}(W \alpha)$, so $J_{r}$ is an ideal of $T$. Clearly $T_{r} \subseteq J_{s}$ if $r \leq s \leq \operatorname{dim} W$, and the containment can be proper. For example, suppose that $s$ is finite and $u \in V \backslash W$. Write $W=\left\langle w_{i}\right\rangle$ with $|I|=s$ and $V=\left\langle v_{p}\right\rangle \oplus\left\langle u, w_{i}\right\rangle$. Let $1 \in I$ and $J=I \backslash\{1\}$, and note that

$$
\alpha=\left(\begin{array}{ccc}
\left\{v_{p}, w_{1}\right\} & w_{j} & v \\
0 & w_{j} & w_{1}
\end{array}\right) \in J_{s} \backslash T_{s} .
$$

More generally, let $Y=\left\{\alpha \in J_{r}: \operatorname{dim}(W \alpha)<\operatorname{dim}(V \alpha)\right\}$. Since $\operatorname{dim}(W \alpha)<r$ for all $\alpha \in J_{r}$, we know that $r(Y) \leq r$. Suppose that $r(Y)<r$. If $r$ is finite then the $\alpha$ defined in (1) with $s=r(Y)$ satisfies $r(Y)=\operatorname{dim}(W \alpha)$, hence it belongs to $J_{r}$. However, it also satisfies $\operatorname{dim}(W \alpha)<\operatorname{dim}(V \alpha)$, so it contradicts the choice of $r(Y)$, and we conclude that $r(Y)=r$. Likewise, if $r$ is infinite, we write $W=\left\langle w_{i}, w_{j}\right\rangle$ where $|I|=r(Y)<r \leq|J|$ and let $V=\left\langle v_{q}\right\rangle \oplus\left\langle w_{i}, v+w_{j}\right\rangle$. Now consider

$$
\alpha=\left(\begin{array}{ccc}
v_{q} & w_{i} & v+w_{j} \\
0 & w_{i} & w_{j}
\end{array}\right) \in J_{r} .
$$

Since this also contradicts the choice of $r(Y)$, we again conclude that $r(Y)=r$. Therefore $J_{r}=T_{r} \cup K(Y)$ by Theorem 11 .

Recall that, for any vector space $U$, the ideals of $T(U)$ form a chain under containment. The next result shows that $T(V, W)$ is almost never isomorphic to any $T(U)$.

COROllary 12. If $\operatorname{dim} V \geq 3$, then $T(V, W)$ is not isomorphic to $T(U)$ for any vector space $U$.

PROOF. By our assumption at the start, $\operatorname{dim} W \geq 1$ and $W \neq V$.

Suppose that $\operatorname{dim} W=1$. In this case, $\operatorname{codim} W \geq 2$ and we can write $V$ $=\left\langle v_{1}, v_{2}, v_{m}\right\rangle \oplus\left\langle w_{1}\right\rangle$ where $W=\left\langle w_{1}\right\rangle$. Define nonzero $\pi_{1}, \pi_{2} \in T(V, W)$ by

$$
\pi_{1}=\left(\begin{array}{cc}
\left\{v_{m}, v_{2}, w_{1}\right\} & v_{1} \\
0 & w_{1}
\end{array}\right), \quad \pi_{2}=\left(\begin{array}{cc}
\left\{v_{m}, v_{1}, w_{1}\right\} & v_{2} \\
0 & w_{1}
\end{array}\right) .
$$


Let $Y_{1}=\left\{\pi_{1}\right\}$ and $Y_{2}=\left\{\pi_{2}\right\}$. If $\beta \in K\left(Y_{1}\right)$ then $\operatorname{ker} \beta \supseteq \operatorname{ker} \pi_{1} \supseteq W$. Hence, if $\lambda \in T(V, W)$ then $V \lambda \beta \subseteq W \beta=\{0\}$, so $\lambda \beta=0 \in K\left(Y_{1}\right)$. That is, $K\left(Y_{1}\right)$ and $K\left(Y_{2}\right)$ are ideals of $T(V, W)$, where $\pi_{1} \in K\left(Y_{1}\right) \backslash K\left(Y_{2}\right)$ and $\pi_{2} \in K\left(Y_{2}\right) \backslash K\left(Y_{1}\right)$. In other words, $K\left(Y_{1}\right)$ and $K\left(Y_{2}\right)$ are ideals of $T(V, W)$ which are not comparable under containment, so the ideals of $T(V, W)$ do not form a chain in this case.

Now suppose that $\operatorname{dim} W \geq 2$. If $w_{1}, w_{2} \in W$ are linearly independent, and $u$ $\in V \backslash W$, then $v_{1}=u+w_{1}$ and $v_{2}=u+w_{2}$ are linearly independent in a complement of $W$ in $V$. Write $V=\left\langle v_{m}\right\rangle \oplus\left\langle w_{n}\right\rangle$, where $\left\{v_{m}\right\}=\left\{v_{1}, v_{2}\right\} \dot{\cup}\left\{v_{p}\right\}$ and $\left\{w_{n}\right\}=\left\{w_{1}, w_{2}\right\} \dot{\cup}\left\{w_{q}\right\}$. Define nonzero $\alpha, \beta \in T(V, W)$ by

$$
\alpha=\left(\begin{array}{ccc}
\left\{v_{p}, w_{n}\right\} & v_{1} & v_{2} \\
0 & w_{1} & w_{2}
\end{array}\right), \quad \beta=\left(\begin{array}{cc}
\left\{v_{m},\left\{w_{n}\right\} \backslash\left\{w_{1}\right\}\right\} & w_{1} \\
0 & w_{1}
\end{array}\right) .
$$

Clearly, $\alpha \in J_{1} \backslash T_{2}$ and $\beta \in T_{2} \backslash J_{1}$. That is, $J_{1}$ and $T_{2}$ are ideals which are not comparable under containment, so the ideals of $T(V, W)$ do not form a chain, and the result follows.

It is not hard to see that part (b) of the next result also holds if $Y=\alpha T^{1}$, which clearly also equals $\beta T^{1}$ for some $\beta \neq \alpha$. So, it is unlikely that there are conditions which determine precisely when $T_{r} \cup K(Y)$ is principal.

THEOREM 13. Let $\alpha \in T(V, W)=T$, say. Then:

(a) $T \alpha T=T_{r} \cup K(Y)$, where $Y=T \alpha T, r=r(Y)=s^{\prime}$ and $s=\operatorname{dim}(W \alpha)$;

(b) $T^{1} \alpha T^{1}=T_{r} \cup K(Y)$, where $Y=\{\alpha\}, r=r(Y)=s^{\prime}$ and $s=\operatorname{dim}(W \alpha)$.

PROOF. (a) Let $s=\operatorname{dim}(W \alpha)$ and, with our usual choice of bases, write

$$
\alpha=\left(\begin{array}{ccc}
u_{i} & w_{j} & v_{k} \\
0 & w_{j}^{\prime} & w_{k}
\end{array}\right)
$$

Let $V=\left\langle u_{\ell}^{\prime}\right\rangle \oplus\left\langle w_{j}^{\prime}\right\rangle \oplus\left\langle w_{k}^{\prime}\right\rangle$ and define $\delta, \varepsilon \in T$ by

$$
\delta=\left(\begin{array}{cc}
\left\{u_{i}, v_{k}\right\} & w_{j} \\
0 & w_{j}
\end{array}\right), \quad \varepsilon=\left(\begin{array}{cc}
\left\{u_{\ell}^{\prime}, w_{k}\right\} & w_{j}^{\prime} \\
0 & w_{j}^{\prime}
\end{array}\right) .
$$

Clearly, if $\pi=\delta \alpha \varepsilon$, then $\operatorname{dim}(W \pi)=s$. Now, if $\lambda, \mu \in T$ then $\operatorname{dim}(W \lambda \alpha \mu)$ $\leq \operatorname{dim}(W \alpha) \mu \leq \operatorname{dim}(W \alpha)=s$ (note that possibly $\alpha \notin T \alpha T)$. Hence, if $Y=T \alpha T$ and $r=r(Y)$ then $r \geq s^{\prime}$. Suppose that $r>s^{\prime}$. Then, by the definition of $r(Y)$, there exists $\lambda, \mu \in T$ such that $s^{\prime} \leq \operatorname{dim}(W \lambda \alpha \mu) \leq \operatorname{dim}(W \alpha)=s$, which is a contradiction (regardless of whether $s$ is finite or infinite). Hence, $r \leq s^{\prime}$, and equality follows. Next, if $\beta \in K(Y)$ then $\beta=\gamma \mu^{\prime}$ for some $\gamma \in Y$ and $\mu^{\prime} \in T$. That is, $\beta=\lambda \alpha \mu$. $\mu^{\prime}$ for some $\lambda, \mu \in T$ and so $\beta \in T \alpha T$. Moreover, for each $\lambda, \mu \in T, \operatorname{dim}(V \lambda \alpha \mu)$ $\leq \operatorname{dim}(W \alpha) \mu \leq s$. In other words, $K(Y) \subseteq T \alpha T \subseteq T_{s^{\prime}}$. In fact, if $\beta \in T_{s^{\prime}}$ then $r(\beta) \leq s=\operatorname{dim}(W \alpha)$, so $\beta \in T \alpha T$ by Lemma 4. Thus, we obtain $T \alpha T=T_{s^{\prime}} \cup K(Y)$, as required. 
(b) If $\lambda, \mu \in T$ and $\lambda \neq 1$ then $r(\lambda \alpha \mu) \leq \operatorname{dim}(W \alpha) \mu \leq s$, so $\lambda \alpha \mu \in T_{s^{\prime}}$, and clearly $\alpha \mu \in K(Y)$ when $Y=\{\alpha\}$. Thus, $T^{1} \alpha T^{1} \subseteq T_{s^{\prime}} \cup K(Y)$. Conversely, if $\beta \in T_{s^{\prime}}$ then $r(\beta) \leq s=\operatorname{dim}(W \alpha)$, so $\beta \in T \alpha T$ by Lemma 4; and, if $\beta \in K(Y)$ then $\beta \in \alpha T^{1}$ by Lemma 3. Therefore, $T^{1} \alpha T^{1}=T_{S^{\prime}} \cup K(Y)$ where $r(Y)=s^{\prime}$ (since $Y=\{\alpha\}$ ).

In passing, we note that if $1 \leq r \leq \operatorname{dim} W, Y=T_{r}$ and $\beta \in K(Y)$, then $r(\beta)$ $\leq r(\alpha)<r$, so $\beta \in T_{r}$ and thus $K(Y) \subseteq T_{r}$. Also, $r(Y)=r$ since $Q_{r} \subseteq T_{r}$. That is, the ideal $T_{r}$ takes the form $T_{r(Y)} \cup K(Y)$, when $Y=T_{r}$.

\section{Acknowledgements}

The author gratefully acknowledges the support of Centro de Matematica, Universidade do Minho and the Portuguese Foundation for Science and Technology through the research program POCTI, during his visit in March-May 2006. He also thanks Diane Brittlebank and Laurie Sullivan for their hospitality during his visit in January 2007 when this paper was completed.

\section{References}

[1] A. H. Clifford and G. B. Preston, The Algebraic Theory of Semigroups, Mathematical Surveys, No. 7, Volumes 1 and 2 (American Mathematical Society, Providence, RI, 1961, 1967).

[2] S. Nenthein and Y. Kemprasit, 'Regular elements of some semigroups of linear transformations and matrices', Int. Math. Forum 2(1-4) (2007), 155-166.

[3] S. Nenthein, P. Youngkhong and Y. Kemprasit, 'Regular elements of some transformation semigroups', Pure Math. Appl. 16(3) (2005), 307-314.

[4] J. Sanwong, B. Singha and R. P. Sullivan, 'Maximal (minimal) congruences on some semigroups', Acta Math. Sin. (Engl. Ser.), to appear.

[5] W. Sommanee, 'Maximal inverse subsemigroups of some semigroups', MS Thesis, Chiangmai University, Chiangmai, Thailand, 2006.

[6] J. S. V. Symons, 'Some results concerning a transformation semigroup', J. Austral. Math. Soc. Ser. A 19(4) (1975), 413-425.

R. P. SULLIVAN, School of Mathematics and Statistics, University of Western Australia, Nedlands, 6009, Australia e-mail: bob@maths.uwa.edu.au 